\title{
Development of Enabling Technologies for Ku-Band Airborne SATCOM Phased-Arrays
}

\author{
Alfredo Catalani ${ }^{1,2, *} \mathbb{C}$, Giovanni Toso ${ }^{1}$, Piero Angeletti ${ }^{1}$, Mario Albertini ${ }^{3}$ and \\ Pasquale Russo ${ }^{2}$ \\ 1 European Space Agency (ESA-ESTEC), TEC-EFA, 2201AZ Noordwijk, The Netherlands; \\ giovanni.toso@esa.int (G.T.); piero.angeletti@esa.int (P.A.) \\ 2 Formerly with Airbus Italy, 00131 Rome, Italy; lino.russo@st4in.com \\ 3 Airbus Italy, Modem \& Gateway, 00156 Rome, Italy; mario.albertini@airbus.com \\ * Correspondence: alfredo.catalani@esa.int; Tel.: +31-71-565-4047
}

Received: 26 February 2020; Accepted: 13 March 2020; Published: 16 March 2020

check for updates

\begin{abstract}
In the paper the development of a fully electronic transmit-receive phased-array antenna system in Ku-band for aircraft communications via satellite is presented. Particular emphasis has been placed in the improvement of the following key elements: a dual-polarization self-diplexing radiating element, a transmit/receive active module with full polarization agility based on a digital vector modulator and a SiGe multinode MMIC. The optimized antenna elements enable a significant improvement towards the realization of a future affordable commercial product for satellite communications.
\end{abstract}

Keywords: active phased arrays; phased array antennas; MMIC; mobile antenna systems; satellite communications

\section{Introduction}

Broadband connectivity has become essential in everyday life both for business and for entertainment. This, together with the increasing demand of mobile connectivity in locations where terrestrial networks are not available or less efficient, has promoted the development of antennas to be installed on aircrafts, trains, boats and vans to provide interconnection via satellite.

Moving from experimental verification to operational conditions, aeronautical broadband services provided by satellites is today a reality; but to be commercially attractive, the services offered via satellite should have a quality comparable to those available via terrestrial networks, should allow the same type of applications and should be offered at an affordable cost.

Together with the technical issues to be addressed, a main driver is the cost, which comprises the cost of ground segment, which is related to materials, installation (mainly related to the antenna system) and maintenance of the equipment, the cost of the satellite segment and the overall operations of the system.

Focusing on the aeronautical platforms, satellite communication is the only means to provide continuous connectivity over the oceans. Airborne RF communication system applications pose challenging problems to current antenna technology:

- Interactions with the aircraft aerodynamics impacting drag, lift and controllability;

- Influence of the aircraft structure on the antenna performance;

- Electromagnetic Compatibility (EMC)/Electromagnetic Interference (EMI) with other RF and/or electronic systems on board the aircraft;

- Very wide-angle scanning performance, so as to operate at the extreme latitudes required by polar routes; 
- Compatibility with other satellite systems and with terrestrial communication services (involving beamwidths, beam pointing accuracy and sidelobes performance);

- Keyhole problem typical of narrow beam antenna systems employing two axes for tracking a satellite. The keyhole is caused either by mechanical constraints or position slew rate limitations of the antenna mount [1].

The ideal antenna should actually be high gain, broadband, low profile, highly reliable and low cost. Different choices of the antenna architecture [2] could bring to a fully mechanical [3], fully electronic [4] or "hybrid" (mechanical-electronic) solutions [5,6].

The fully mechanical and hybrid solutions are based on antennas with rectangular or highly elliptical apertures in order to minimize the antenna thickness. They create highly elliptical beams and, because they rely usually only on two motors, it is not always possible to project the most directive cut of the pattern along the equatorial plane. The addition of a third axis permits limiting this keyhole problem. Solutions based on fully electronic steering solve the keyhole issue and permit to maintain a transmit-receive link also when flying near the Equator where respecting the regulatory aspects is particularly challenging. Moreover, the fully electronic antenna, thanks to the graceful degradation (typical of active arrays) exhibits lower costs in terms of maintenance as compared to mechanically steering antennas. Electronically scanning antennas can be flat or conformal. In this development conformal antenna are considered in order to guarantee equalized performances in an entire half sphere. It is important to mention that by limiting the scanning directions with respect to the zenith within a cone with an angular opening of 60 degrees instead of 90 degrees, the antenna thickness could be reduced by a factor of two.

\section{Antenna Requirements}

The main requirements for the Ku-band aeronautical market, in terms of antenna characteristics, are summarized in Table 1.

Table 1. Antenna operative requirements.

\begin{tabular}{lc}
\hline \multicolumn{1}{c}{ ITEM } & Value \\
\hline Frequency band-RF received signal & $10.7-12.75 \mathrm{GHz}$ \\
Frequency band-RF transmitted signal & $14.0-14.5 \mathrm{GHz}$ \\
Polarization-RF received signal & Dual orthogonal linear or dual orthogonal circular \\
Polarization-RF transmitted signal & Single adjustable linear \\
\hline
\end{tabular}

The operational band is the complete Ku-Band $(10.7-12.75 \mathrm{GHz}$ in receive and $14.0-14.5 \mathrm{GHz}$ in transmit). The realignment of the polarization in respect to the satellite for both receiver and transmit signals, with the capability to operate also with the circular polarization in receiver mode, is required.

The minimum antenna requirements in terms of operative field of view (e.g., antenna gain-to-noise temperature $[\mathrm{G} / \mathrm{T}]$ over RF band in the entire field of view and for every selectable polarization, Equivalent Isotropic Radiated Power [EIRP] over RF band in the entire field of view and cross-polar discrimination (including pointing error)) are reported in the Table 2.

Table 2. Antenna performance requirements.

\begin{tabular}{lc}
\hline \multicolumn{1}{c}{ ITEM } & Value \\
\hline Radiation gain pattern & In agreement with [7] \\
\hline Field of view & $0^{\circ}<\phi<360^{\circ}$ \\
& $0^{\circ}<\theta<90^{\circ}$ \\
\hline G/T & $>8 \mathrm{~dB} / \mathrm{K}$ \\
\hline EIRP & $>43 \mathrm{dBW}$ \\
\hline
\end{tabular}


Table 2. Cont.

\begin{tabular}{lc}
\hline \multicolumn{1}{c}{ ITEM } & Value \\
\hline Number of beams & 1 \\
\hline Cross polarization discrimination & $>15 \mathrm{~dB}$ \\
\hline
\end{tabular}

It has to be underlined that an antenna in transmission mode has to respect the applicable regulations [7] for the EIRP antenna patterns expressed in terms of power density per bandwidth as shown in Figure 1.

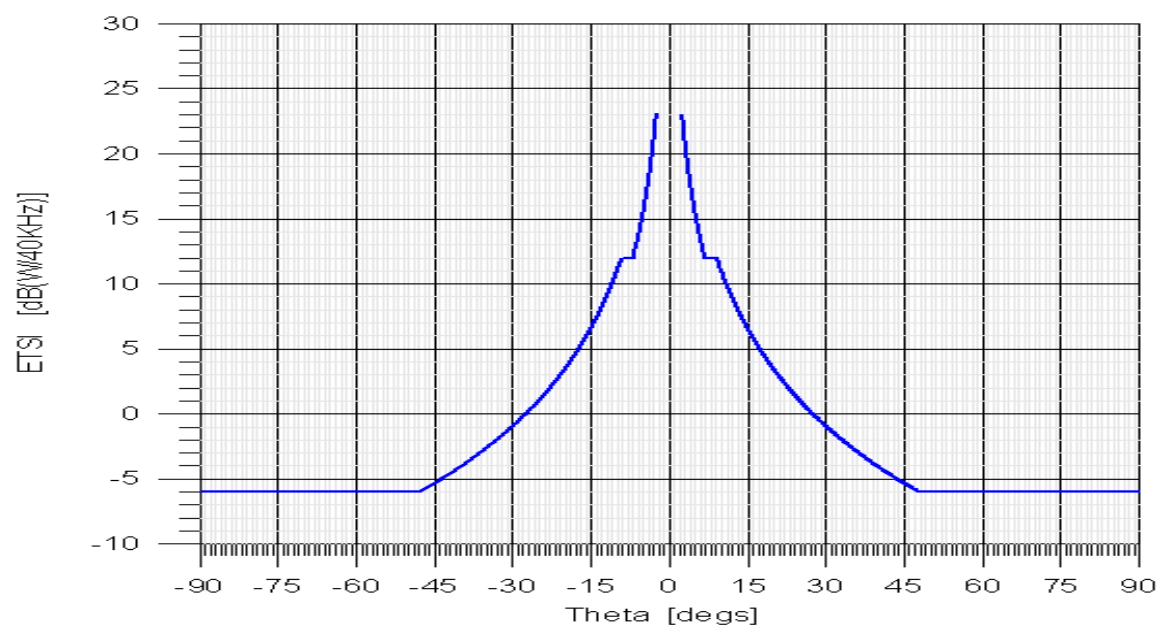

Figure 1. European Telecommunications Standards Institute (ETSI) regulation mask [7].

Moreover, complexity and cost of the antenna are other key factors to take into account together with practical feasibility and ability to operate simultaneously in receiver and transmit modes.

\section{Antenna Design}

The hemi-spherical antenna array when mounted on the aircraft is able to guarantee the proper link, whatever the position of the airborne with respect to the satellite is, thanks to its shape [8]. In order to minimize the complexity of the antenna, the hemi-spherical continuous surface has been approximated with flat facets. To replace the sphere, the icosahedrons, a polyhedron with twenty equilateral triangles, has been selected as starting geometry. Then a new solid of 80 facets has been generated; where, to the original vertexes, the points of middle edge of each triangle have been added after the projection on the sphere surface. Half portion of the new solid is covered by sub-arrays and, after further refinements, the position of the tile is adjusted to avoid the intersection of the extruded part which contains the RF components and the control logic. As a result of the optimization, the geodesic hemi-sphere with 40 facets shown in Figure 2 has been selected as antenna aperture. Each triangular face is composed by the same sub-array, i.e., the same number of radiating elements arranged in a triangular lattice.

The final optimized antenna is able to combine on the same aperture the receiver and transmitter capabilities, has a diameter of $84 \mathrm{~cm}$, a height of $39 \mathrm{~cm}$ and a weight in the order of $90 \mathrm{Kg}$. The number of radiating elements is 1440 with an inter-spacing of $22 \mathrm{~mm}$, which corresponds to one wavelength in the transmit higher frequency. Each triangular sub-array, indicated as tile, presents 36 radiating elements.

For a fixed beam direction, only a sector composed by 14 tiles is active while all the other elements are turned off. All the radiating elements in the active sector are excited in order to generate an equi-phase wave front perpendicular to the desired beam direction. 
It is important to note that when projecting the conformal aperture in an arbitrary pointing direction, the triangles are deformed and become irregular; this concept is clear watching at Figure 2. The resulting sparse array, thanks to this irregular lattice, exhibits a pattern without grating lobes. Moreover, the optimization of the excitation coefficients in amplitude and phase, or in phase only, permits to maintain the side lobes within the spectral density mask imposed by the regulatory aspects as demonstrated in the paper.

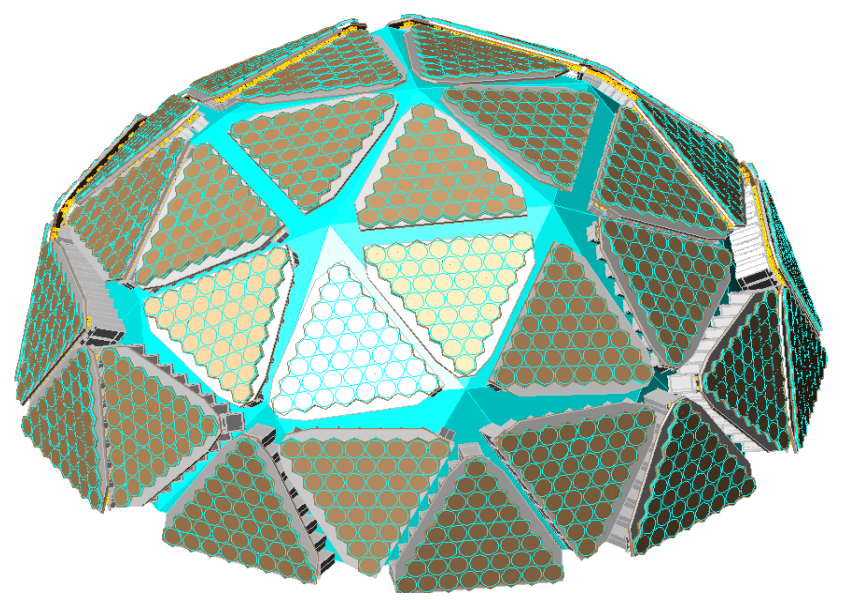

Figure 2. Faceted hemi-spherical antenna.

Concerning the antenna electrical architecture, the input/output ports are fed with the IF band signal, and converted in a Ku band signal by the up/down converter. Each tile is composed by the radiating elements and the transmit/receive modules, directly connected on the back. Two dedicated beam forming networks 1:40 for the receiver and the transmitter mode are connected to the tiles and the CPU controls a set of switches in order to involve the selected sub-arrays to form the antenna beam. The CPU controls the beam steering and the polarization alignment.

The tracking capability of the antenna permits to point the beam electronically in a hemi-spherical field of view (i.e., 360 degrees in azimuth and 90 degrees in elevation) with negligible degradations of the electrical performance, guaranteeing a minimum G/T of $8 \mathrm{~dB} / \mathrm{K}-1$ and an EIRP of $43 \mathrm{dBW}$.

The results obtained using the simulated pattern of an embedded radiating element are reported in the following. The excitation coefficients have been optimized considering the realistic functionality of the Receive(Rx)/Transmit(Tx) active module (combining the amplitude and phase of the two orthogonal polarization for each radiating element).

The G/T result is shown in Figure 3a. The performance matches the required $8 \mathrm{~dB} / \mathrm{K}$ in the entire field of view. As expected, the degradation of the performance at lower pointing angles is due to the different orientation angles of the tiles, which are involved in the beam forming, with respect to the horizon.

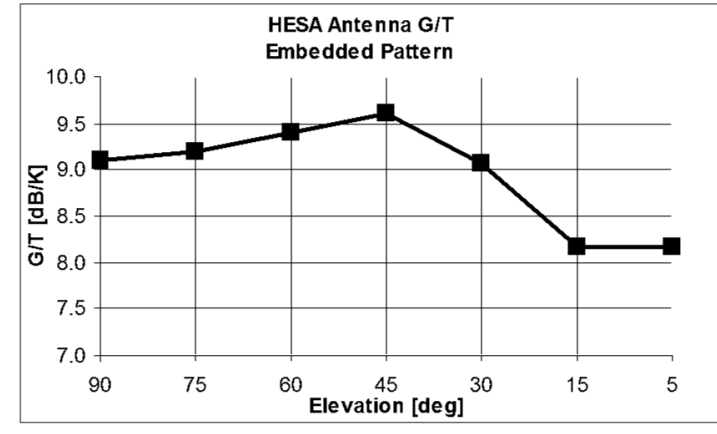

(a)

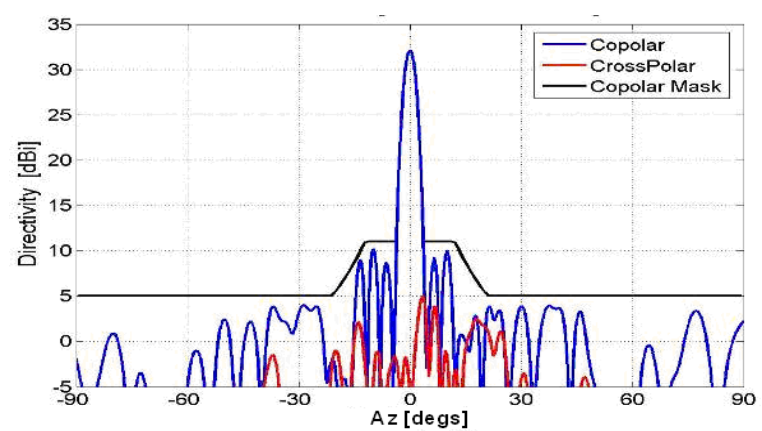

(b)

Figure 3. G/T vs. elevation angle over the horizon (a) and typical azimuth Rx radiation pattern (b). 
The typical azimuth receiver antenna pattern is shown in Figure 3b. During the transmission, all the active devices are able to work at the maximum power level and the expected performance in term of EIRP are reported Figure 4: The minimum guaranteed value is in the order of $43 \mathrm{dBW}$ over the complete antenna field of view.

The antenna is also able to control the transmit antenna patterns side-lobe in order to respect the applicable regulatory recommendations such as ETSI in the European countries [7] and the FCC in the United States.

The typical azimuth transmit antenna pattern is reported in Figure 5a. The black line represents the mask that has been imposed in the optimization process (corresponding to the ETSI mask for 512 $\mathrm{KHz}$ bandwidth). The corresponding elevation transmit antenna pattern is shown in Figure $5 \mathrm{~b}$.

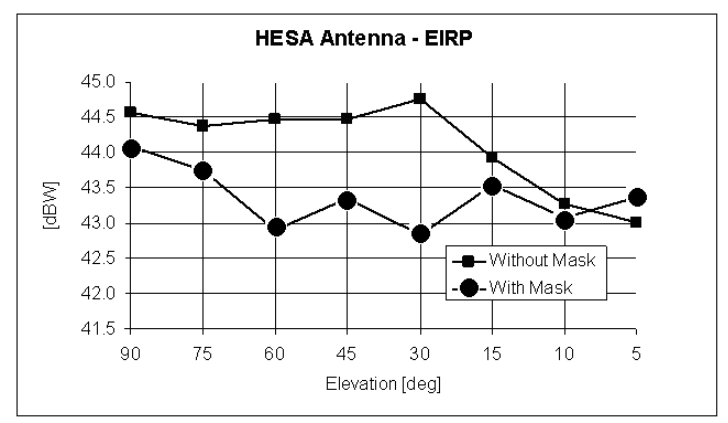

Figure 4. EIRP vs. elevation angle over the horizon (with and without mask constraint).

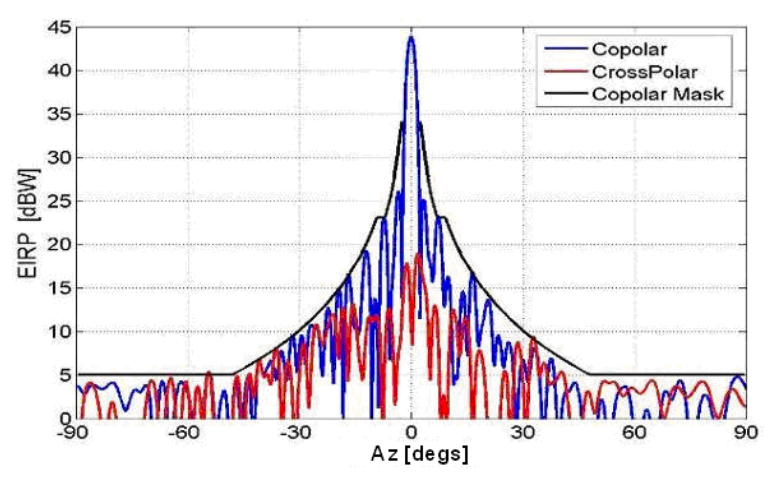

(a)

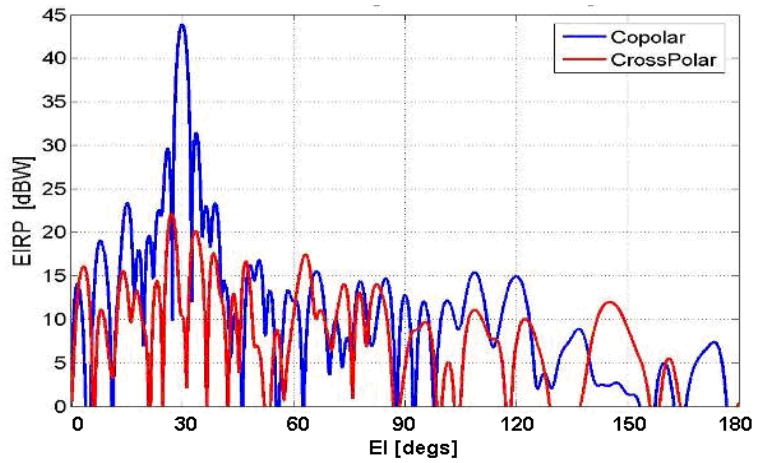

(b)

Figure 5. Typical azimuth (a) and elevation (b) Tx antenna pattern at $14.25 \mathrm{GHz}, 30$ degrees elevation over the horizon.

\section{Developed Devices}

The main challenges in the design of the antenna have been: (a) the development of the radiating element, (b) the development of the active module for transmitter/receiver and (c) the design and manufacturing of a digital vector modulator (DVM) able to integrate in a single chip the attenuator and phase shifter features. These three components are described in the following paragraphs.

\subsection{Self-Diplexing Radiating Element Design}

The first criticality in the design of $\mathrm{T} x / \mathrm{Rx}$ radiators is the capability to separate the transmitted signal from the received one. A typical approach consists in designing a radiating element covering the entire band and then connecting a diplexer or a circulator. However, this configuration has the drawback to increase the overall dimensions and the ohmic losses at the Low Noise Amplifier (LNA) input. 
A more efficient solution consists of implementing, for each radiating element, two different ports: One for Tx and one for Rx signal. This type of configuration is called a self-diplexing solution. This approach offers better electrical performance and permits the reduction of the manufacturing complexity.

The radiating element is realized using two different substrates of RT/Duroid 5880 with $0.508 \mathrm{~mm}$ thickness separated by a foam with $1 \mathrm{~mm}$ thickness.

Starting from the ground, the first two metallizations are dedicated to the receive capabilities: The first patch has a diameter of $8.5 \mathrm{~mm}$ and it is fed by a UT047 coaxial cable; while the second patch, with a diameter of $10.4 \mathrm{~mm}$, implements the stacked configuration, by the foam spacer, that allows to operate over the large frequency band. The transmit radiating configuration uses a single patch of $7.4 \mathrm{~mm}$ diameter and it is fed directly by UT047 coaxial cable taking advantage of the upper receive metallization for the ground. The Figure 6a shows a sketch of the self-diplexing radiating element assembly; while in Figure 6b the 3D model used in full wave analysis is shown.

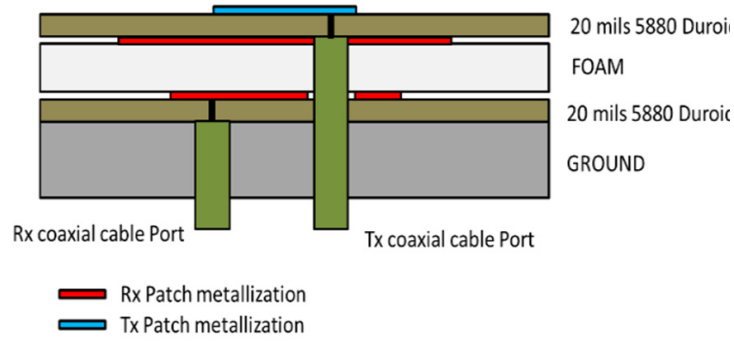

(a)

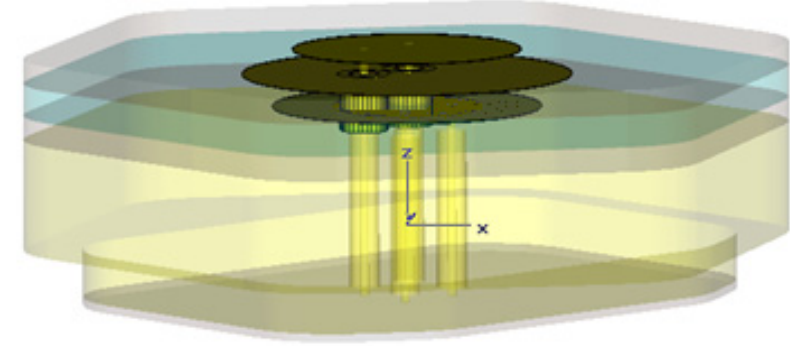

(b)

Figure 6. Sketch of the self-diplexing radiating element (a) and 3D model (b).

The radiating element provides four ports able to operate in dual linear orthogonal polarizations both for transmission and reception.

A prototype of the radiating element is shown in Figure $7 \mathrm{a}$, while the engineered radiator is shown in Figure 7b. The hexagonal ground makes support functionality and it circumscribes a circle of $22 \mathrm{~mm}$ of diameter.

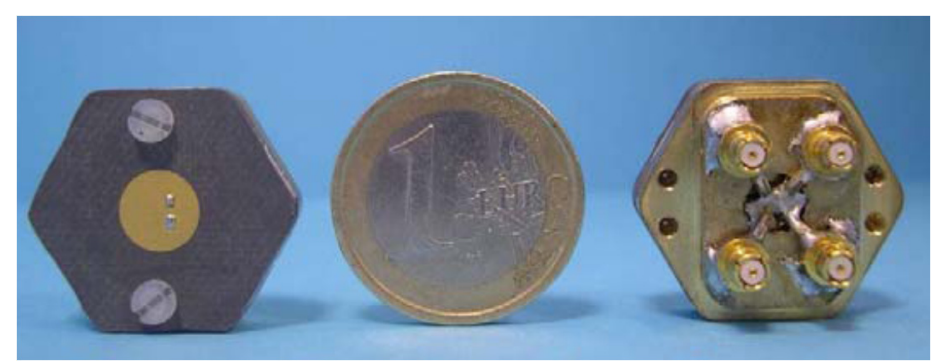

(a)

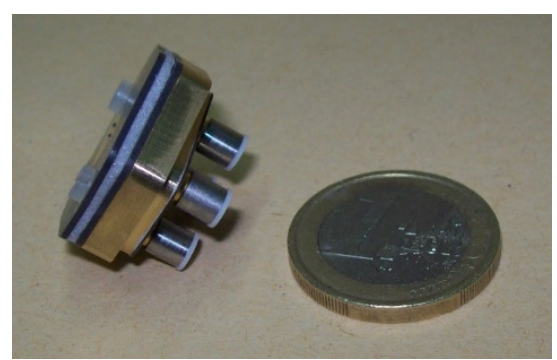

(b)

Figure 7. Radiating element prototype (a) and engineered radiator (b).

Return loss at Rx and Tx ports and isolation are reported in Figures 8 and 9, respectively. It is important to note that the isolation between the Rx and Tx ports over the entire Tx band is better than $20 \mathrm{~dB}$.

The gain for the Rx and Tx radiating elements have been measured vs. frequency. The results are reported in Figure 10a,b. The gain in free space condition is better than $8 \mathrm{~dB}$ for the receive ports over the frequency band with VSWR of 2:1; while at transmit ports $7 \mathrm{~dB}$ gain are guaranteed. 


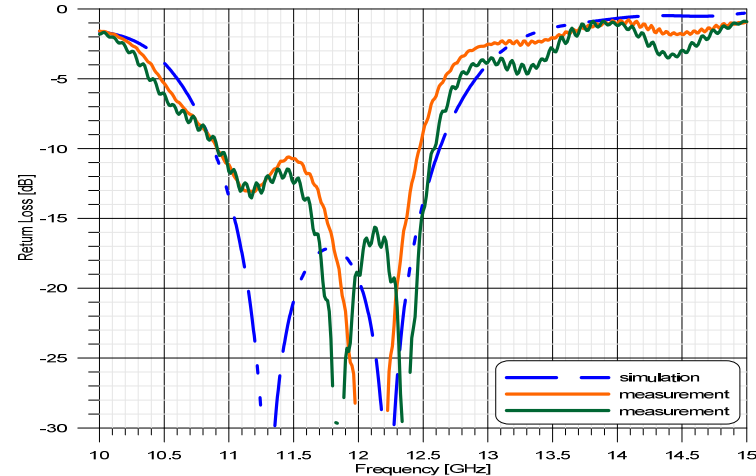

(a)

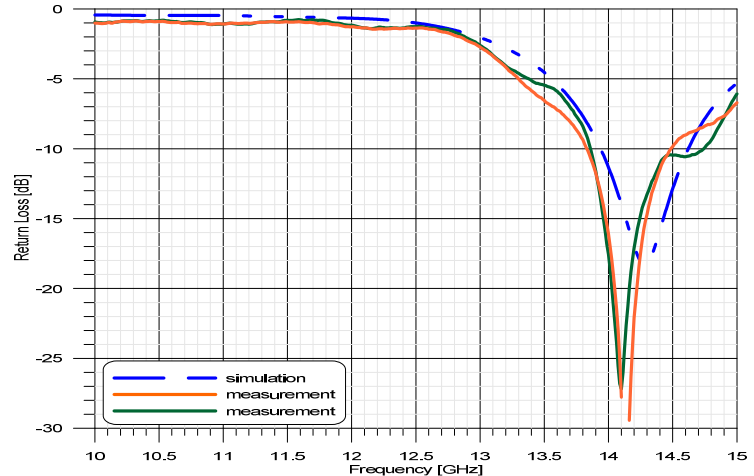

(b)

Figure 8. Return loss of the radiating element (simulation and measurements) at Rx ports (a) and Tx ports (b).

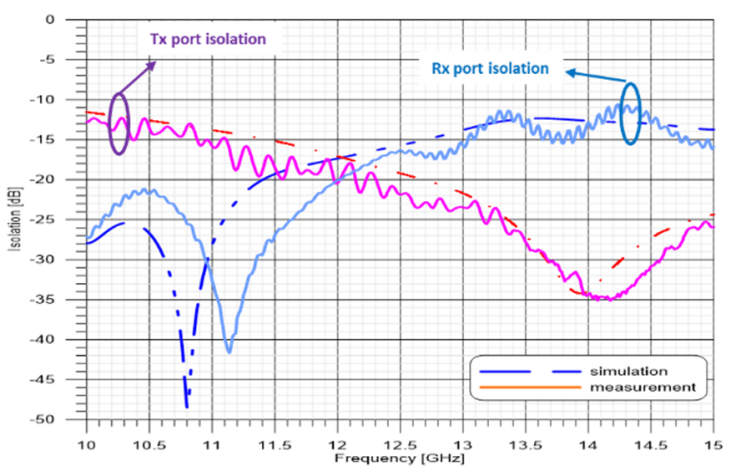

(a)

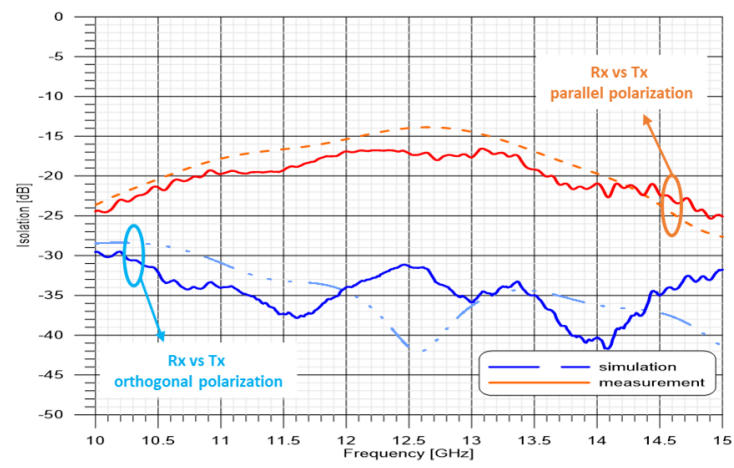

(b)

Figure 9. Isolations of the radiating element (simulation and measurements): (a) at $R \times 1 \mathrm{vs}$. $R \times 2$ and Tx1 vs. Tx2; (b) Rx ports vs. Tx ports.

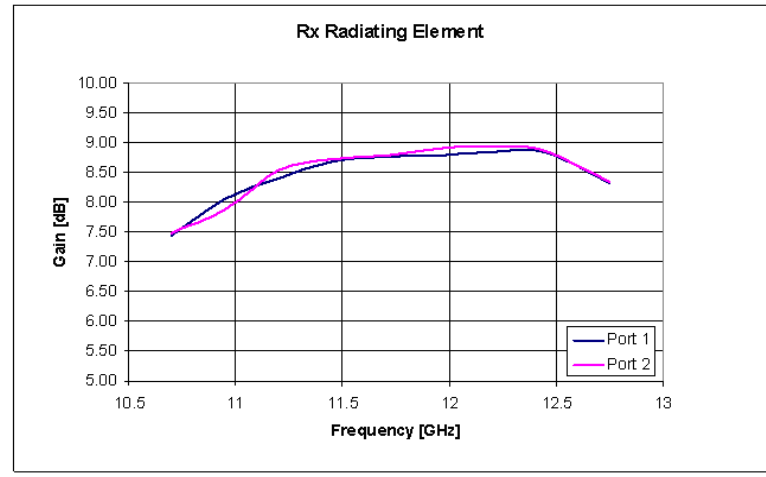

(a)

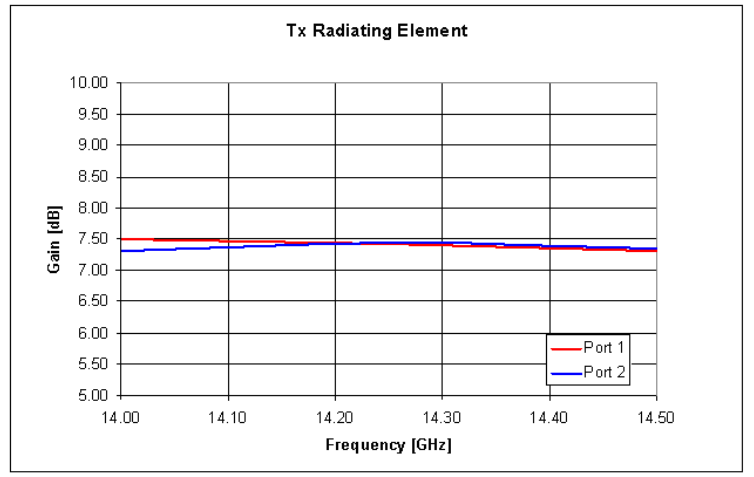

(b)

Figure 10. Measured gain: (a) Rx radiating element; (b) Tx radiating element.

In Figure 11, the measured radiation patterns of the radiating element in free space configuration are reported for the extreme frequencies of the operating receive band, while for the transmitting band it shows the central frequency in the same conditions. The reported measured radiation patterns are compared with the simulation analysis results demonstrating an excellent agreement. 


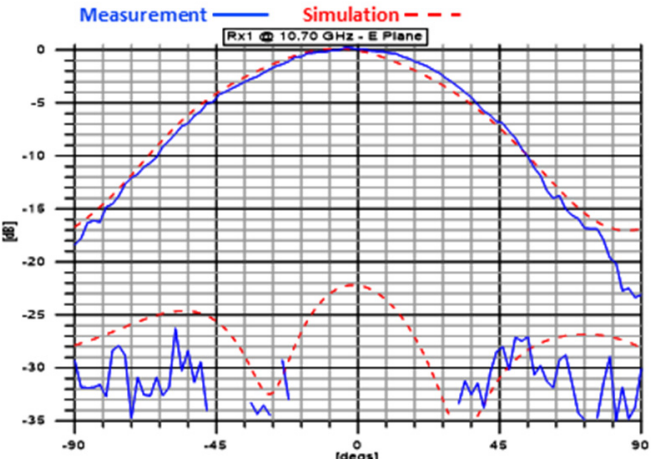

(a) E-plane @10.7 GHz

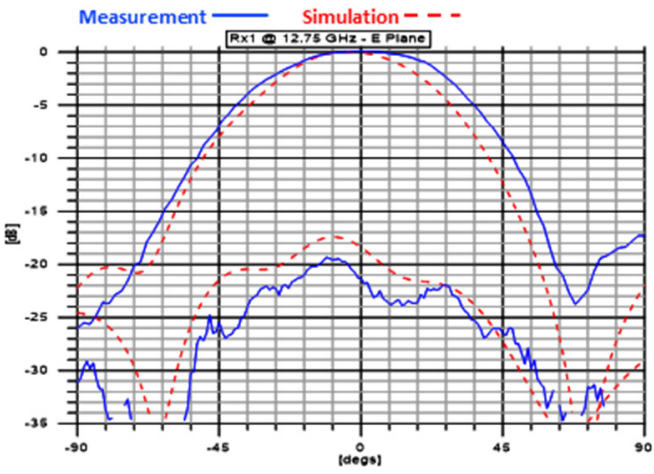

(c) E-plane @12.75 GHz

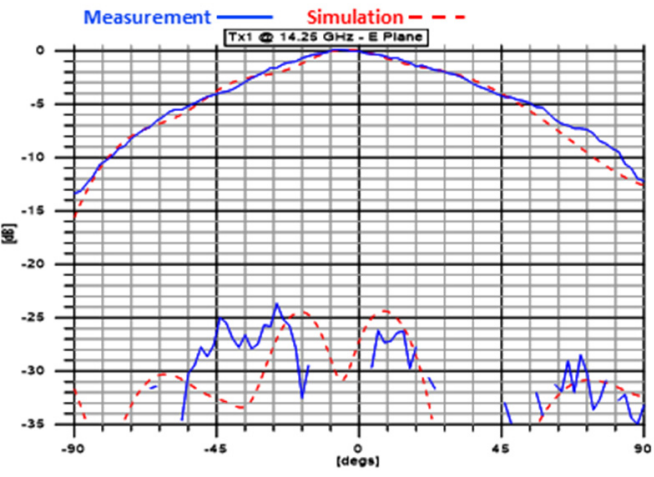

(e) E-plane @14.25 GHz

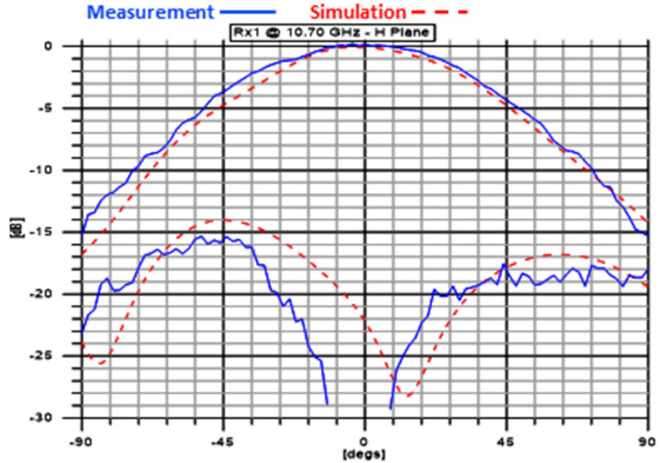

(b) H-plane @10.7 GHz

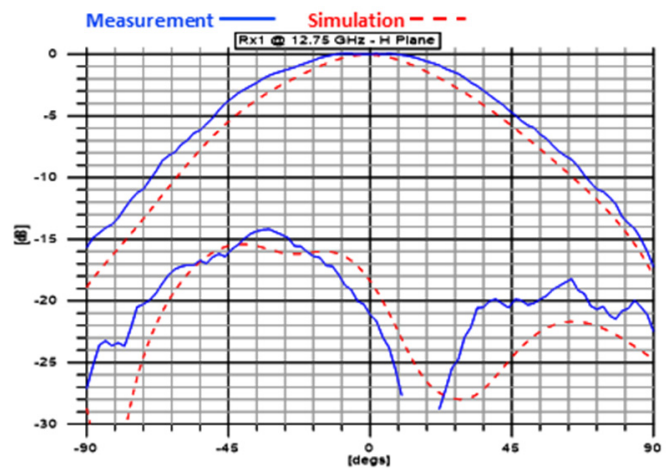

(d) H-plane @12.75 GHz

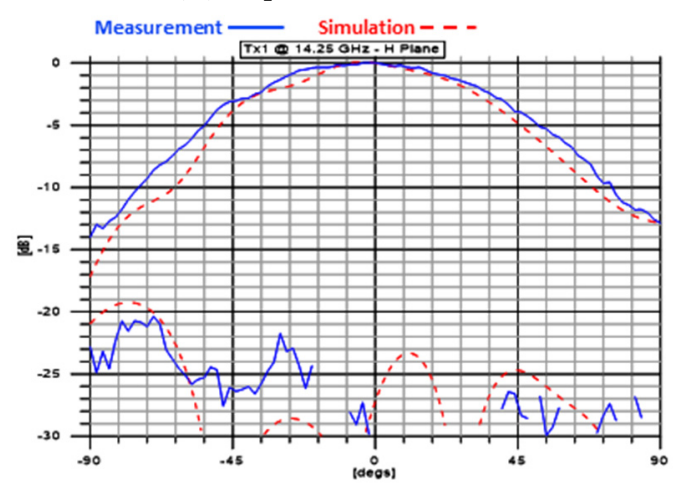

(f) H-plane @14.25 GHz

Figure 11. E-plane and $\mathrm{H}$-plane radiation patterns for $\mathrm{Rx}$ at $10.7 \mathrm{GHz}(\mathbf{a})$ and (b) and at $12.75 \mathrm{GHz}$ (c) and (d); for Tx at $14.25 \mathrm{GHz}$ (e) and (f). Continuous blue line: measured results, dashed red line: numerical simulations.

\subsection{Transmit/Receive Active Module Design}

Each radiating element is directly connected with an active circuitry that provides the functionality of the Tx/Rx module. The Tx/Rx module (intellectual property of Airbus Italy [9]) is able to realign the linear polarization in receive and in transmit or, alternatively, may allow the generation of a circular polarization. The phase shifters used for the polarization control are also able, at the same time, to form the antenna beam imposing the correct relative phase delay between the elements. The active modules are composed by the receive device and the transmit device (the assembled $\mathrm{Tx} / \mathrm{Rx}$ device is shown in Figure 12).

The manufactured modules are presented in Figure 13: In particular, the control board sides are shown. Their dimensions are 20.6 by 7 by $37 \mathrm{~mm}^{3}$, with a weight of $15 \mathrm{~g}$. 


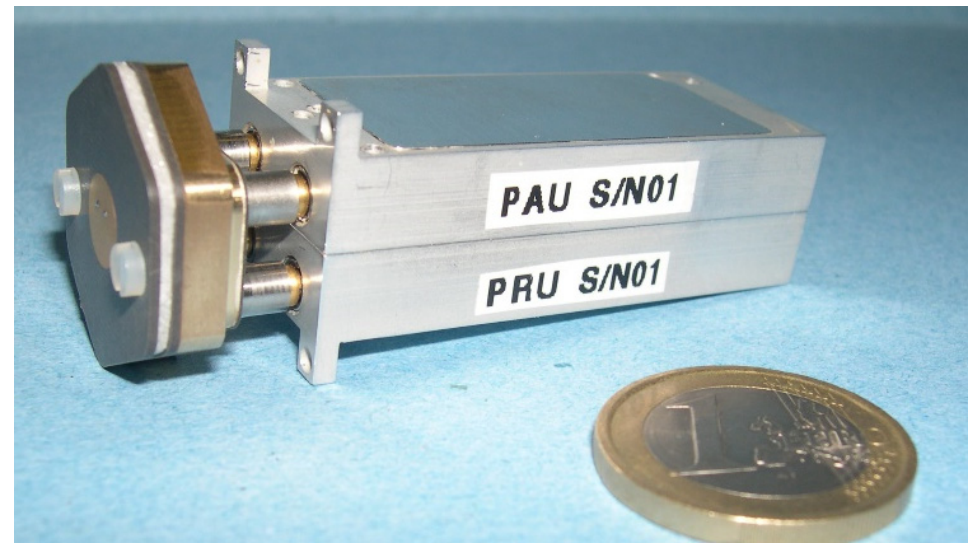

Figure 12. $\mathrm{Tx} / \mathrm{Rx}$ active module connected to the radiating element.

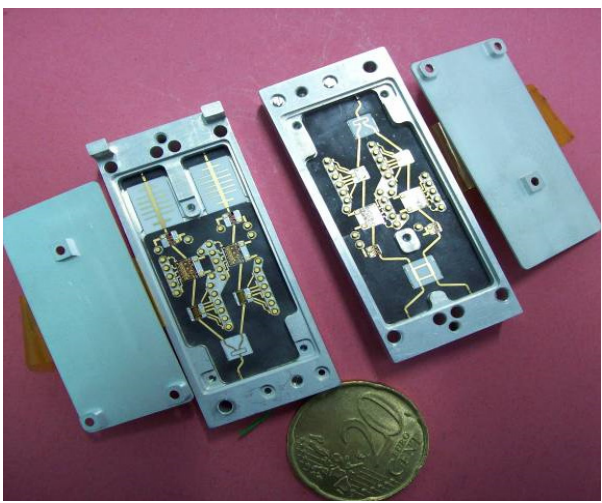

(a)

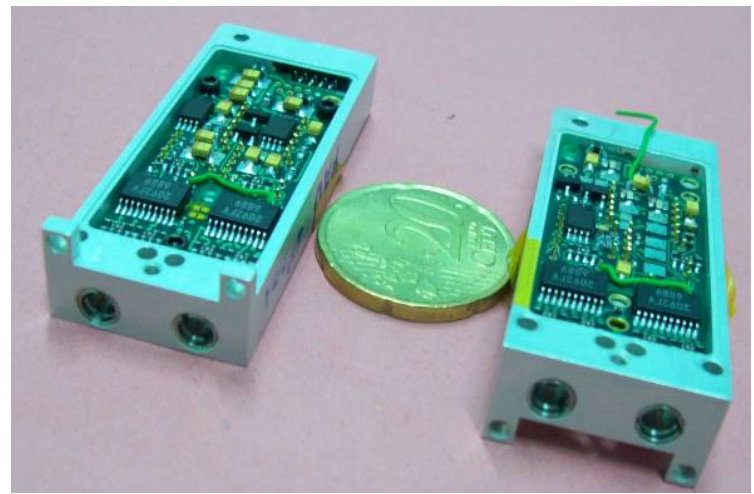

(b)

Figure 13. Tx/Rx active module: (a) Tx and Rx RF active board; (b) Tx and Rx digital control board.

Figure 14a shows the channel gain behavior vs. the frequency when the attenuator and the phase shifter are set at the nominal value (zero). Figure 14b shows how all the SSPAs (Solid State Power Amplifier) power is moved between the two output ports when the relative phase between the two channels is properly set: The correct rotation of the linear polarization is provided by the opportunely balanced power.

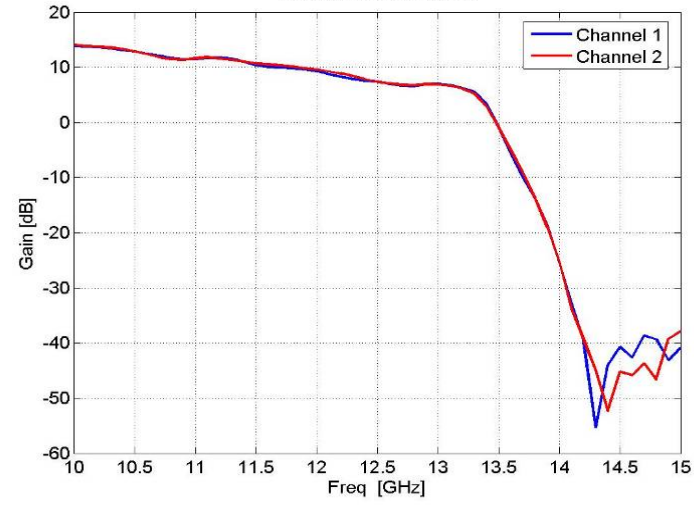

(a)

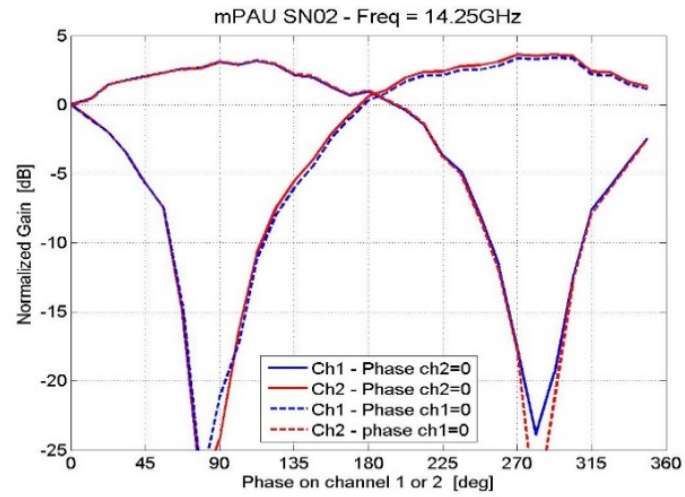

(b)

Figure 14. Tx/Rx active module performance: (a) Rx active module measured gain; (b) Tx active module control power allocation measured between the two output ports. 


\subsection{Digital Vector Modulator (DVM)}

A DVM in Ku band to control in amplitude and phase the radiating elements has been designed, manufactured and tested. It consists of a MMIC (Monolithic Microwave Integrated Circuit) in GaAs (Gallium Arsenide) technology integrating a 5-bit attenuator (ATT), a 5-bit phase shifter (PS) and a serial to parallel converter (S2P), as reported in Figure 15.

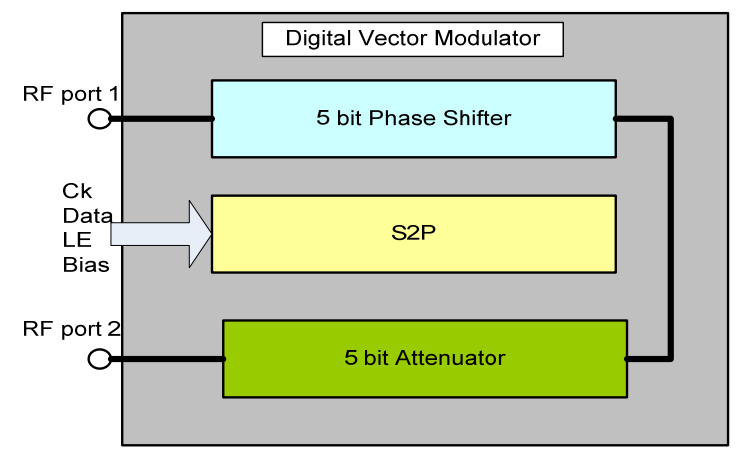

Figure 15. GaAs digital vector modulator (DVM) MMIC.

The DVM development allows to realize T/R Modules in Ku band with mechanical dimensions and interconnections reduced with respect to that available on the market, i.e., separated digital attenuator and phase shifter (Figure 16).

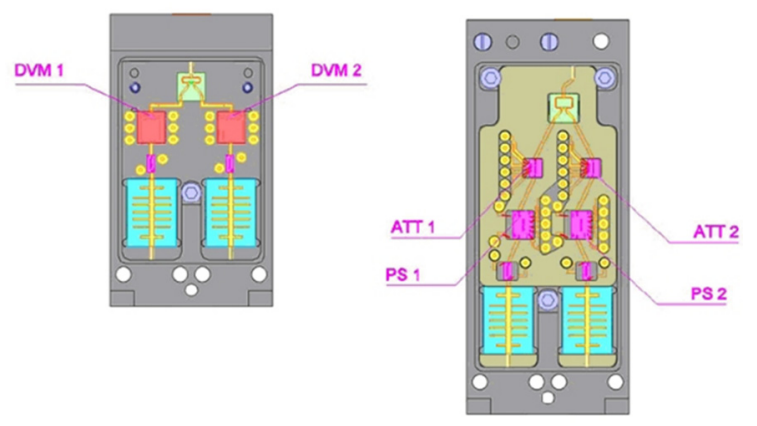

Figure 16. Rx module with (left) and without (right) DVM.

To realize a S2P on GaAs technology the E-mode and the D-mode transistors should be realized on the same wafer. To do this, the TQPED (MMIC technological process) process has been selected. A 10 bit S2P has been customized by TriQuint in line with Airbus Italy requirements summarized in Table 3 .

Table 3. Basic characteristics of the DVM.

\begin{tabular}{ll}
\hline \multicolumn{1}{c}{ ITEM } & Value \\
\hline Phase Shifter Cells & $180^{\circ}, 90^{\circ}, 45^{\circ}, 22.5^{\circ}, 11.25^{\circ}$ \\
Attenuator Cells & $0.75,1.5,3,6,12 \mathrm{~dB}$ \\
Rx Band & $10.7 \div 12.75 \mathrm{GHz}$ \\
Tx Band & $14 \div 14.5 \mathrm{GHz}$ \\
Command Line & Clock, Data, LE \\
Bias & $+5 ;-5 \mathrm{~V}$ \\
Power Consumption & $<350 \mathrm{~mW}$ \\
I/O Return Losses & $>12 \mathrm{~dB}$ \\
\hline
\end{tabular}

Each attenuator and phase shifter cell have been designed separately. The position of the cells in the MMIC chain has been properly chosen considering the RF performances and the layout arrangement. 
The DVM MMIC and the stand alone main block have been manufactured on a 5 by $10 \mathrm{~mm}^{2}$ tile in a multi project wafer (MPW). The manufactured MMIC has been mounted on an aluminum carrier and tested by means of a probe station. The pictures of the DVM under microscope is reported in Figure 17.

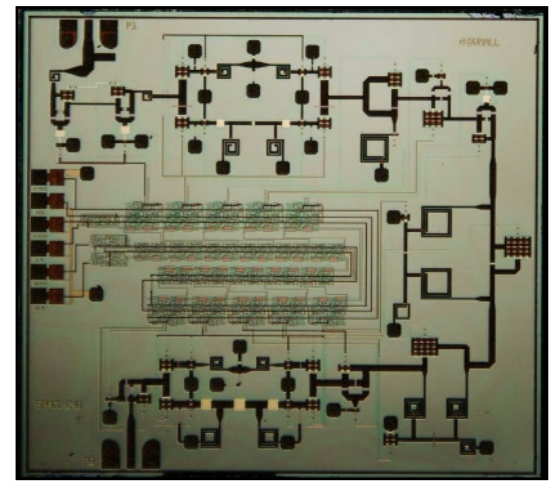

Figure 17. picture under microscope of the manufactured DVM.

The RF measurements are hereafter provided: Relative amplitude and phase variations (Figure 18), measured insertion loss with only phase shifter variation and phase error due to the attenuation states (Figure 19), return loss port 1 and return loss port 2 (Figure 20).

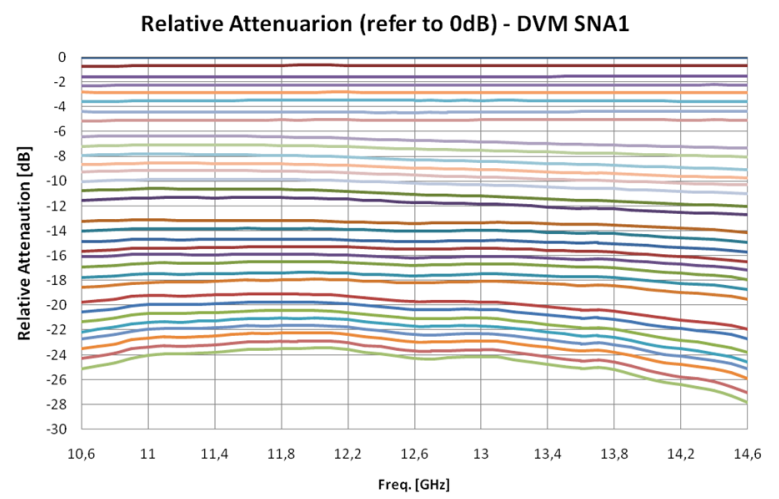

(a)

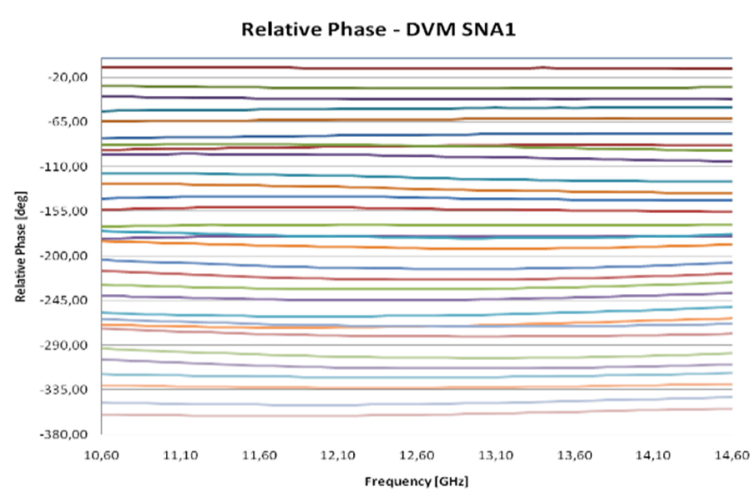

(b)

Figure 18. DVM chip performance: (a) measured relative attenuation with $1 \mathrm{~dB}$ step; (b) measured relative phase with 11.25 degrees step.

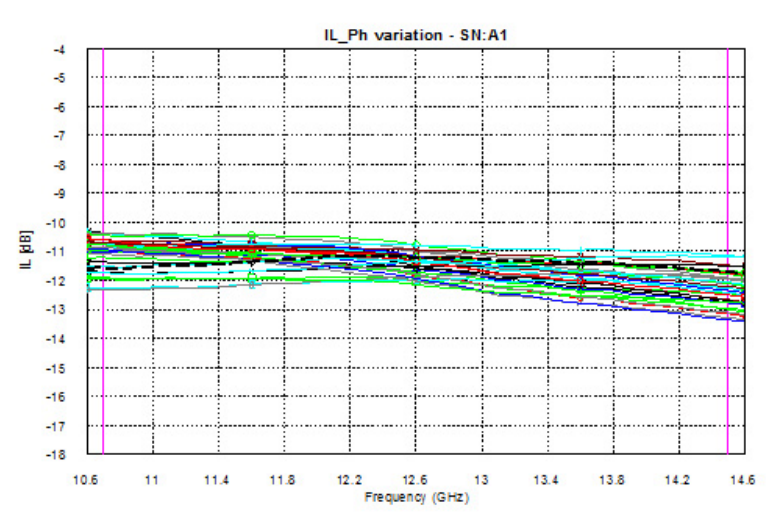

(a)

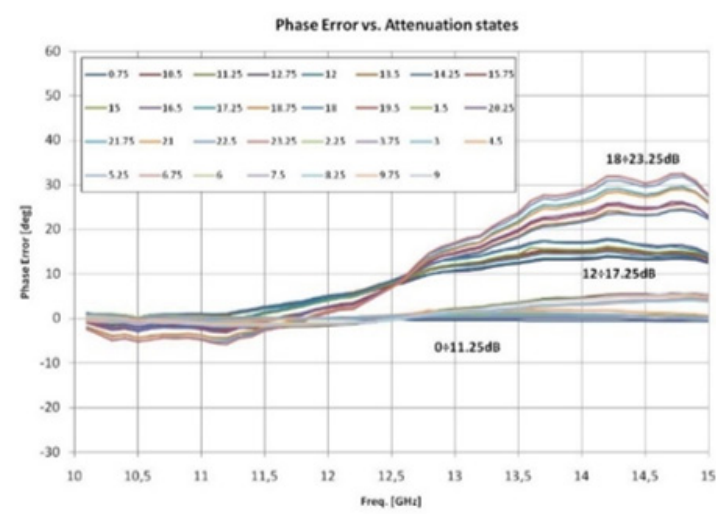

(b)

Figure 19. DVM chip transmission performance: (a) measured insertion loss-only phase shifter variation (32 states); (b) phase error due to the attenuation states, refer to $0 \mathrm{~dB}$ and 0 phase state. 


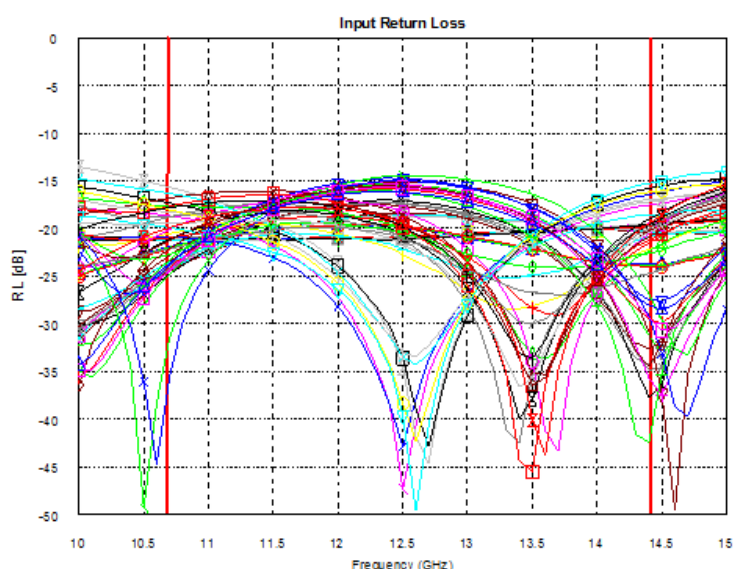

(a)

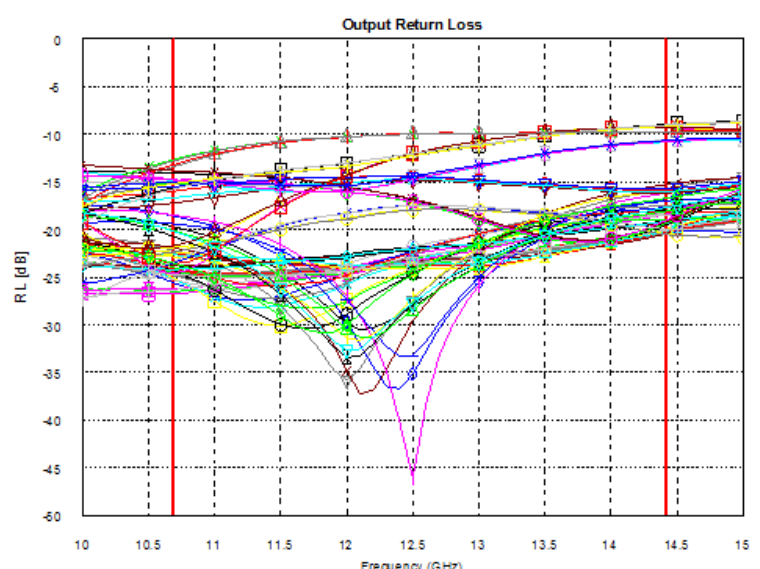

(b)

Figure 20. DVM chip return loss performance: (a) port 1; (b) port 2.

The performances of the $11.25^{\circ}, 90^{\circ}$ and $180^{\circ}$ phase shifter cells in the DVM MMIC were very good in terms of relative phase shift: An average error of $1^{\circ}$ has been measured. Vice versa, the measurement of the $22.5^{\circ}$ and $45^{\circ}$ cells shows a phase shift higher than the target value $\left(8^{\circ}\right.$ and $4^{\circ}$, respectively). It has to be highlighted that the phase errors of the $22.5^{\circ}$ and $45^{\circ}$ cells in the stand alone main blocks is the half respect the same cells inside the DVM MMIC.

The performance of the attenuator cells agree with the expected ones, except for the phase stability vs. gain variation in Tx band which presented an undesired phase shift for attenuation higher than $17.25 \mathrm{~dB}$. However, such deviation for the specific parameter is generally also present in commercial devices and is not considered significant for the specific project.

The insertion loss of the reference state was about $11.2 \mathrm{~dB}$ and flat in band. Considering the combination of the two COTS (Commercial of-the-shelf) component employed in the antenna demonstrator we experienced an overall insertion loss of $13 \mathrm{~dB}$. Taking into account the inductive effect of the wire bonding interconnections, the return losses of both ports are below $10 \mathrm{~dB}$.

Finally, the power consumption of the S2P, designed by TriQuint, was $250 \mathrm{~mW}$. This could be drastically reduced (up to $120 \mathrm{~mW}$ ) with the implementation of some identified technical solutions.

\section{Test Campaign Results}

The activity has been finalized with an exhaustive test campaign dedicated to evaluate first, the performance of the single radiating element in active configuration (Figure 21), second, considering it as a demonstrator of a linear sub-array of 5 active radiating elements embedded within the complete tile composed by 36 dummy radiators in triangular lattice (Figure 22).

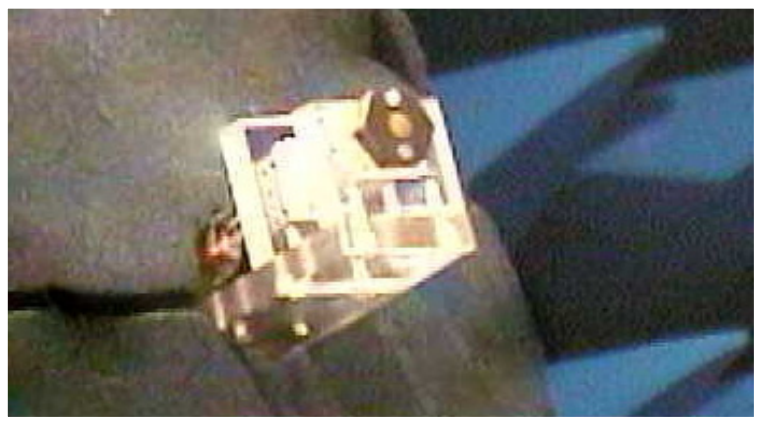

Figure 21. Active radiating element in free space configuration. 


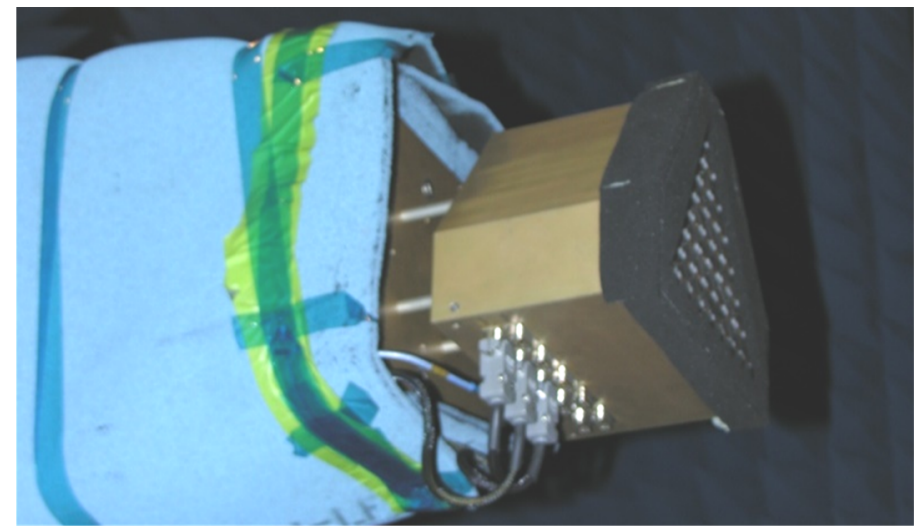

Figure 22. Active antenna demonstrator.

The radiation pattern of the active radiating element in free space configuration have been measured in several cases where the impinging RF wave in linear polarization was characterized by a defined alignment angle with respect to the radiating element axes. Setting the proper amplitude and phase coefficients, the active radiating element was able to align correctly the received polarization providing a very good discrimination between the two orthogonal polarizations. Figure 23a reports the received cross polar component over the complete operating band, when the attenuator and the phase shifter controls are correctly set for a copolar signal with the polarization rotated by 155 degrees; while Figure 23b shows the same behavior for the transmit channel over the entire frequency band.

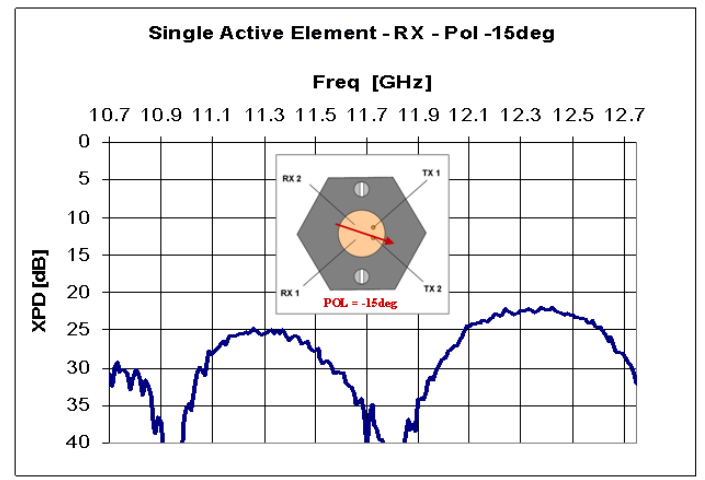

(a)

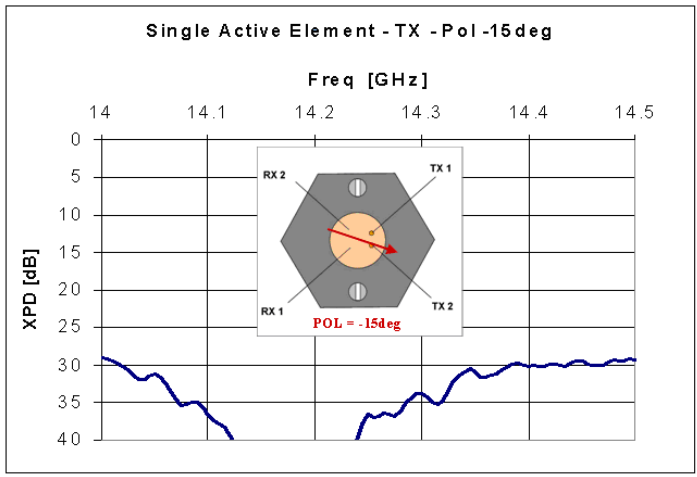

(b)

Figure 23. Example of cross polarization discrimination over the frequency band: (a) Rx; (b) Tx.

Figure 24a shows the measured radiation pattern in receive when a misalignment of 30 degrees has been corrected by the Tx/Rx Active Module.

The same performance has been provided for the active radiating element operating in transmit mode: Figure $24 \mathrm{~b}$ shows an example of radiation pattern for a transmitted polarization rotated of 15 degrees with respect to the nominal axes.

Figure 22 shows the manufactured antenna demonstrator which implements the triangular array geometry in the real dimensions and representing the tile of the hemi-spherical phased array shown in Figure 2. A subset composed of five radiating elements is connected to the $\mathrm{Tx} / \mathrm{Rx}$ active modules and the radiation pattern has been measured for different beam steering positions and polarization alignments.

Figure 25a shows the measured radiation pattern when the excitation coefficients have been applied to steer the beam at 10 degrees without the recovery of a polarization misalignment (30 degrees); while in Figure $25 \mathrm{~b}$ it reported the same measurement but with the recovery of the polarization angle. 


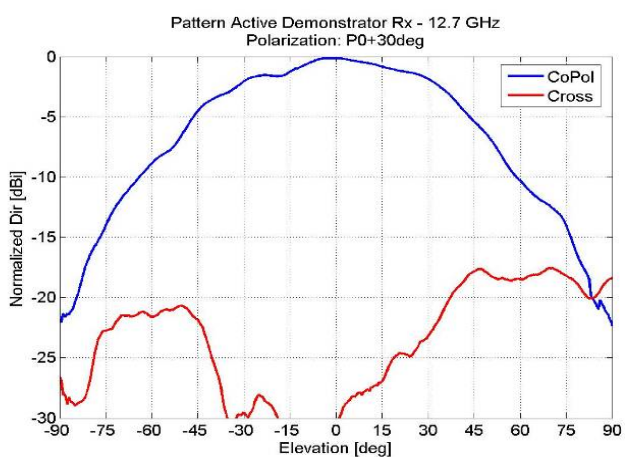

(a)

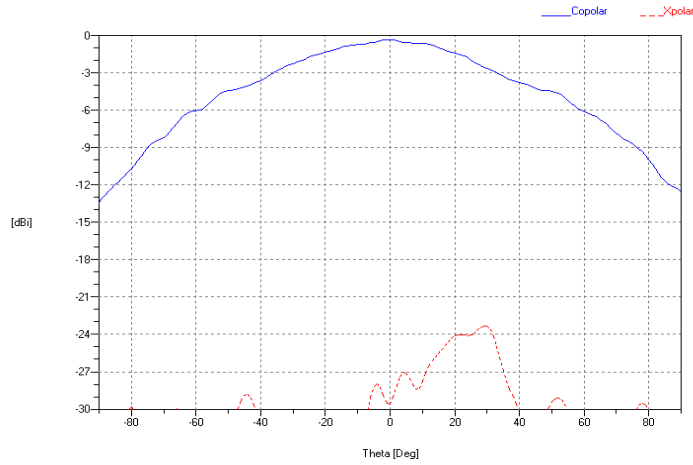

(b)

Figure 24. Measured pattern after polarization alignment: (a) Rx signal (11.7 GHz) impinging with 30 degrees misalignment with the nominal; (b) Tx signal (14.0 GHz) transmitted with 15 degrees polarization rotated.

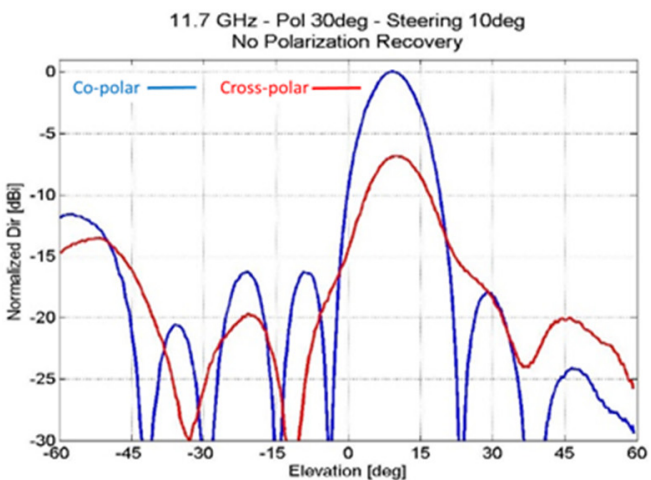

(a)

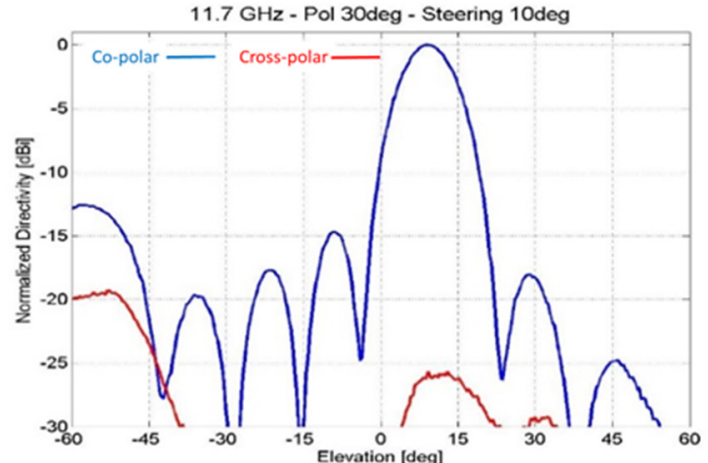

(b)

Figure 25. Measured Rx pattern of the active demonstrator: (a) only steering coefficients applied; (b) steering and polarization recovery coefficients applied.

Figure 26 shows the measured radiation pattern in transmit mode when the excitation coefficients are applied to provide the beam steering of -10 degrees and a polarization rotation of 30 degrees.

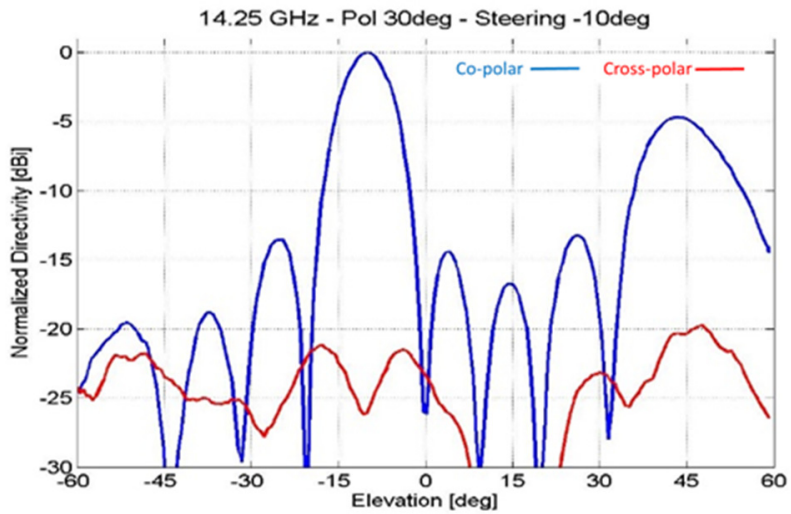

Figure 26. Transmit measured pattern when applying excitation coefficients relevant to $-10^{\circ}$ steering angle and recovering $30^{\circ}$ polarization misalignment.

\section{Conclusions}

The reported technological solutions constitute the base of on-going developments for affordable phased-array antennas for commercial aeronautical satellite communications. Similar developments 
are currently ongoing at Airbus Italy targeting higher frequencies (i.e., Ka-band) as well as space-borne phased-arrays.

Author Contributions: Conceptualization, A.C., P.R. and M.A.; Validation, A.C. and M.A.; Supervision, G.T. and P.A. All authors have read and agreed to the published version of the manuscript.

Funding: This research was funded by ESA/ESTEC ARTES 5.1 "Advanced Antenna Concepts for Aircraft In-Flight Entertainment" project, Contract No. 19508/05/NL/JA.

Conflicts of Interest: The authors declare no conflict of interest.

\section{References}

1. Dunlop, G.; Ellis, P.J.; Afzulpurkar, N.V. The Satellite Tracking Keyhole Problem: A Parallel Mechanism Mount Solution. Trans. N. Z. Inst. Eng. 1993, 20, 1-7.

2. Lisi, M.; Angeletti, P.; Catallo, C.; Florio, A.; Lucchi, G.; Cucci, A. Airborne Antennas for Broadband Satellite Communications. In Proceedings of the 25th ESA Antenna Workshop on Satellite Antenna Technology, Noordwijk, The Netherlands, 18-20 September 2002.

3. Bellaveglia, G.; Marcellini, L.; Ferrarotti, A.; Arenaccio, S.; Forti, R.L. Two-way low profile satellite antenna system for mobile applications. In Proceedings of the 30th ESA Antenna Workshop, Noordwijk, The Netherlands, 27-30 May 2008.

4. Adler, C.O.; Monk, A.D.; Rasmussen, D.N.; Taylor, M.J. Two-way airborne broadband communications using phased array antennas. In Proceedings of the 2003 IEEE Aerospace Conference, Big Sky, MT, USA, 8-15 March 2003; Volume 2, pp. 925-932.

5. Halsema, P.; Anderson, B.; Frisco, J. Airborne DBS Receive System. In Proceedings of the Antennas and Propagation Society International Symposium, Montreal, QC, Canada, 13-18 July 1997; Available online: https://apps.dtic.mil/dtic/tr/fulltext/u2/a355120.pdf (accessed on 1 July 1998).

6. Shelley, M.; Pearson, R.; Vasquez, J. Low Profile, Dual Polarised Antenna for Aeronautical and Land Mobile Satcom. In Proceedings of the 4th Advanced Satellite Mobile Systems (ASMS'08), Bologna, Italy, 26-28 August 2008; pp. 13-15.

7. ETSI EN 302 186 V1.1.1 (2004-01), Satellite Earth Stations and Systems (SES); Harmonized EN for Satellite Mobile Aircraft Earth Stations (AESs) Operating in the 11/12/14 GHz Frequency Bands Covering Essential Requirements under Article 3.2 of the R\&TTE Directive. Available online: https://www.etsi.org/deliver/etsi_ en/302100_302199/302186/01.01.01_60/en_302186v010101p.pdf (accessed on 23 January 2004).

8. Catalani, A.; Paolo, F.D.; Migliorelli, M.; Russo, L.; Toso, G.; Angeletti, P. Ku band hemispherical fully electronic antenna for aircraft in flight entertainment. Int. J. Antennas Propag. 2009, 2009, 230650. [CrossRef]

9. Russo, P.; Catalani, A.; di Paolo, F.; Migliorelli, M. A System for Electronically Aligning the Polarization of an Antenna. U.S. Patent No.7,969,357 B2, 28 June 2011.

(C) 2020 by the authors. Licensee MDPI, Basel, Switzerland. This article is an open access article distributed under the terms and conditions of the Creative Commons Attribution (CC BY) license (http://creativecommons.org/licenses/by/4.0/). 